\title{
RESPUESTA DE LAS PLANTAS DE CASTAÑA AMAZONICA Bertholletia exce/sa A CUATRO NIVELES DE FERTILIZACION CON NITROGENO, FOSFORO Y POTASIO EN LA AMAZONIA PERUANA
}

\author{
Ronald CORVERA-GOMRINGER ${ }^{1}$, Edgar CUSIAUCA ${ }^{1}$ \\ 1 Instituto de Investigaciones de la Amazonía Peruana (IIAP). Programa PROBOSQUES. \\ Jr. Ica Nº 1662, Puerto Maldonado. E-mail: ronaldcorvera@gmail.com.
}

\section{RESUMEN}

En este estudio, se presenta la capacidad de respuesta de Bertholletia excelsa a una aplicación localizada de nutrientes con fuentes de nitrógeno, fósforo y potasio en suelos ácidos con cuatro niveles de urea, superfosfato triple y cloruro de potasio, respectivamente. Los resultados muestran diferencias significativas $(\mathrm{P}<0,01)$ en todas las variables evaluadas, por efecto de la fertilización nitrogenada con niveles de 50 y 100 $\mathrm{kg}$ ha-1. Se observó también respuesta importante con fósforo usando niveles de $200 \mathrm{~kg}$ ha-1 aunque no hubo un efecto estadísticamente significativo.

PALABRAS CLAVE: Bertholletia excelsa, Amazonía peruana, fertilización, macronutrientes.

\section{BRAZILIAN NUT PLANTS, Bertholletia excels, RESPONSE TO FOUR FERTILIZATION LEVELS WITH NITROGEN, PHOSPHORUS AND POTASSIUM IN THE PERUVIAN AMAZON.}

\section{ABSTRACT \\ This study presents the response of Bertholletia excelsa to a localized nutrient supply with sources of nitrogen, phosphorous and potassium on acid soils with four levels of urea, triple superphosphate and potassium chloride, respectively. Significant differences were detected $(\mathrm{P}<0.01)$, in all variables evaluated, by the effect of the nitrogen fertilization using levels of 50 y $100 \mathrm{~kg} \mathrm{ha}^{-1}$. An important, although not a significant effect, was observed with the phosphorus using levels of $200 \mathrm{~kg} \mathrm{ha}^{-1}$.}

KEYWORDS: Bertholletia excelsa, Peruvian Amazon, fertilization, macronutrients. 


\section{INTRODUCCIÓN}

Los nutrientes son distribuidos heterogéneamente en los suelos, debido por ejemplo a la descomposición localizada de la materia orgánica. En cultivos mixtos, que es común encontrar en fincas de agricultura de subsistencia y en sistemas agroforestales, las diferentes especies asociadas pueden variar en la manera de hacer uso de las condiciones heterogéneas del suelo (Emmerich, 1997).

Los suelos en la Amazonía peruana presentan una serie de limitaciones de orden químico que le confieren una fertilidad natural baja. Sin embargo, existen especies que pueden prosperar bajo estas condiciones. La castaña Amazónica Bertholletia excelsa puede ser considerada como una especie que ha logrado evolucionar y prosperar en lugares específicos.

La castaña es una especie de gran porte, que está distribuida en bosques de tierra firme de la Amazonía. Es una especie de particular importancia en aspectos sociales y económicos para las familias rurales que viven principalmente de la explotación de sus rodales naturales. Algunas investigaciones fueron realizadas en esta especie abordándose aspectos de su distribución espacial (Peres \& Baider, 1997), demografía (Zuidema \& Boot, 2002), regeneración y establecimiento de plántulas (Kainer et al., 1998; Zuidema et al., 1999; Myers et al., 2000; Peña-Claros et al., 2002). También estudiaron aspectos de su fenología, polinización y dispersión (Nelson \& Sabih, 1985; Peres et al., 1997; Maués \& Oliveira, 1999; Corvera-Gomringer et al., 2010;); así como también se realizaron estudios de su genética poblacional (Pardo, 2001; O'Maley et al., 1988, Reátegui-Zirena et al., 2009). Pero hasta el momento, poco se sabe sobre la relación entre la productividad de las castañas y otros factores que podrían ser manejados para favorecer la producción, y consecuentemente favorecer el desempeño económico en los sistemas extractivos. Este estudio pretende contribuir a este conocimiento a través la evaluación de la capacidad de respuesta de cuatro niveles de fertilización de nitrógeno, fósforo y potasio localizados en suelos con plantas de Bertholletia excelsa.

\section{MATERIAL Y MÉTODOS}

El estudio se realizó en el Centro Experimental "Fitzcarrald" del Instituto de Investigaciones de la Amazonía Peruana (Coord. UTM E: 464476; N: 8600219), ubicado a $21 \mathrm{Km}$ de la ciudad de Puerto Maldonado, Región Madre de Dios, al 1 sud este de la Amazonía peruana.
Los aspectos generales del sitio se pueden describir como terraza alta, de acuerdo al mapa ecológico del Perú (ONERN, 1972), corresponde a la zona de vida Bosque Húmedo Subtropical (según L. Holdridge, 1967), cuya altitud se encuentra a 220 m.s.n.m. Posee una precipitación de $1986 \mathrm{~mm}$ anuales, una temperatura media de $26^{\circ} \mathrm{C}$ y una humedad relativa de $80 \%$.

La topografía predominante es casi plana, con suelos de buen y moderado drenaje. Son suelos de reacción fuertemente ácida $(\mathrm{pH}$ 4.5-5.5), y provistos medianamente de materia orgánica solo en el horizonte $A_{1}$. Los contenidos de fósforo y potasio disponibles son bajos o en cantidades inadecuadas (2.9 ppm y $48 \mathrm{ppm}$, respectivamente). Un elemento que es perjudicial para las plantas es el aluminio y el porcentaje de saturación de este catión fluctúa entre 50 y $70 \%$.

Los suelos de la zona son de textura franco arenosos, bien drenados, superficiales y poco profundos. Son clasificados como Tropudults (USA Soil Taxonomy); Acrisoles (FAO, 1988). Son suelos de origen aluvial antiguo que presentan un buen desarrollo a través del perfil, con presencia de arcilla iluvial en los "peds" del horizonte B.

Por lo general los suelos de la región presentan limitantes de orden químico, ya que son de reacción ácida, con un contenido bajo de materia orgánica, bajo en fósforo disponible, bajo en potasio disponible y capacidad de intercambio catiónico baja (Corvera-Gomringer et al., 2010). Estas características le confieren un grado de fertilidad natural baja, siendo necesaria la aplicación de dosis de correctivos y fertilizantes, si se quiere resultados satisfactorios en la producción.

El experimento se inició el mes de Mayo del 2012, cuando las plantas de Bertholletia excelsa fueron embolsadas con sustrato enriquecido e instaladas en el terreno definitivo. Las bolsas - $20 \mathrm{~cm}$ de diámetro y $31 \mathrm{~cm}$ de longitud - fueron separadas a $40 \mathrm{~cm}$ de distancia.

Las fuentes y dosis utilizadas fueron las siguientes: nitrógeno (Urea), fósforo $\left(\mathrm{P}_{2} \mathrm{O}_{5}\right)$ y potasio $\left(\mathrm{K}_{2} \mathrm{O}\right)$ a razón de: (1) sin fertilización (testigo con CIC 6.40); (2) $50 \mathrm{~kg}$ ha-1; (3) $100 \mathrm{~kg} \mathrm{ha}^{-1}$; (4) $200 \mathrm{~kg}$ ha-1 y (5) $400 \mathrm{~kg}$ ha-1 en una sola aplicación (Tabla 1). El diseño experimental fue de Bloques al azar con tres repeticiones.

Las variables evaluadas en los muestreos no destructivos fueron las siguientes: altura de la planta, diámetro basal, número de hojas y longitud de la primera hoja verdadera. En este trabajo se analizaron los resultados correspondientes a cuatro muestreos (Junio - Septiembre), para cada una de las variables, como indicador de la tendencia general del experimento. 
Para determinar los incrementos en altura y diámetro de las plantas de Bertholletia los datos experimentales fueron sometidos a la prueba de normalidad de Shapiro Wilk, análisis de varianza y prueba de significancia de Tukey $(\mathrm{P}<0.01)$ utilizando el programa estadístico SAS (SAS, Institute, NC, 2001) e Infostad.

\section{RESULTADOS}

Se identificaron diferencias significativas $(\mathrm{P}<0.01)$ para las variables de estructura de la planta, como altura y diámetro basal. En las evaluaciones de altura de la planta (Tabla 2) el tratamiento $3(20.50 \mathrm{~cm})$, resultó con el mejor incremento y con diferencias significativas $(\mathrm{P}<0.01)$ a los tratamientos $1(6.18$ $\mathrm{cm}), 2(8.03 \mathrm{~cm}), 6(8.70 \mathrm{~cm}), 7(3.50 \mathrm{~cm}), 8(13.67$ $\mathrm{cm}), 9(10.3 \mathrm{~cm}), 10(4.83 \mathrm{~cm}), 11(3.17 \mathrm{~cm})$ y 12 $(5.17 \mathrm{~cm})$. Los tratamientos $4(1.00 \mathrm{~cm}), 5(0.00 \mathrm{~cm})$ y $13(2.17 \mathrm{~cm})$ mostraron los valores más bajos y fueron diferentes al resto de los tratamientos (Figura 1).
Para el incremento en diámetro basal de la planta (Tabla 3) los tratamientos $2(0.24 \mathrm{~cm}), 3(0.24 \mathrm{~cm})$ y $8(0.24 \mathrm{~cm})$ fueron los que resultaron con el mejor incremento al compararse con los tratamiento restantes, sin embargo, no se encontraron diferencias significativas entre ellos (Figura 2).

Comparando todos los tratamientos del estudio para determinar los incrementos de altura y diámetro estadísticamente significativos, se sugiere que la mejor respuesta de absorción de macro nutrientes fue la del nitrógeno con dosis de $100 \mathrm{~kg} \mathrm{ha}^{-1}$. Sin embargo, cuando las dosis de nitrógeno fueron mayores (200 y $\left.400 \mathrm{~kg} \mathrm{ha}^{-1}\right)$ se evidenció un efecto severo de toxicidad, ocasionando la muerte de la planta con la aplicación de la dosis máxima.

Para el caso de los tratamientos con dosis de fósforo $\mathrm{y}$ potasio los incrementos en altura y diámetro generalmente no fueron significativos, exceptuando el tratamiento 8 (200 $\mathrm{kg} \mathrm{ha}^{-1}$ de fósforo) que representó el segundo mejor incremento en altura y en diámetro.

Tabla 1. Cantidades netas de nutrimentos aplicados en los diferentes tratamientos (kg ha-1).

\begin{tabular}{cccc}
\hline Tratamiento & N & $\mathbf{P}$ & $\mathbf{K}$ \\
\hline & 0 & 0 & 0 \\
\hline T1 & -------- \\
T2 & 50 & 0 & 0 \\
T3 & 100 & 0 & 0 \\
T4 & 200 & 0 & 0 \\
T5 & 400 & 0 & 0 \\
T6 & 0 & 50 & 0 \\
T7 & 0 & 100 & 0 \\
T8 & 0 & 200 & 0 \\
T9 & 0 & 400 & 0 \\
T10 & 0 & 0 & 50 \\
T11 & 0 & 0 & 100 \\
T12 & 0 & 0 & 200 \\
T13 & 0 & 0 & 400 \\
\hline
\end{tabular}

${ }^{*}$ El tratamiento 1 (testigo) no recibió fertilización 
Tabla 2. Incremento de altura en plantas de castaña Bertholletia excelsa. Prueba de Tukey.

\begin{tabular}{cccc}
\hline Tratamiento & Medias & $\mathbf{n}$ & Significancia \\
\hline T3 & 20.50 & 3 & A \\
T8 & 13.67 & 3 & A, B \\
T9 & 10.43 & 3 & A, B \\
T6 & 8.70 & 3 & A, B \\
T2 & 8.03 & 3 & A, B \\
T1 & 6.18 & 9 & A, B \\
T12 & 5.17 & 3 & A, B \\
T10 & 4.83 & 3 & A, B \\
T7 & 3.50 & 3 & A, B \\
T11 & 3.17 & 3 & A, B \\
T13 & 2.17 & 3 & B \\
T4 & 1.00 & 3 & B \\
T5 & 0.00 & 3 & B \\
\hline
\end{tabular}

Alfa:0.01 DMS: $=17.41574$

Letras distintas indican diferencias significativas $(p<=0.01)$

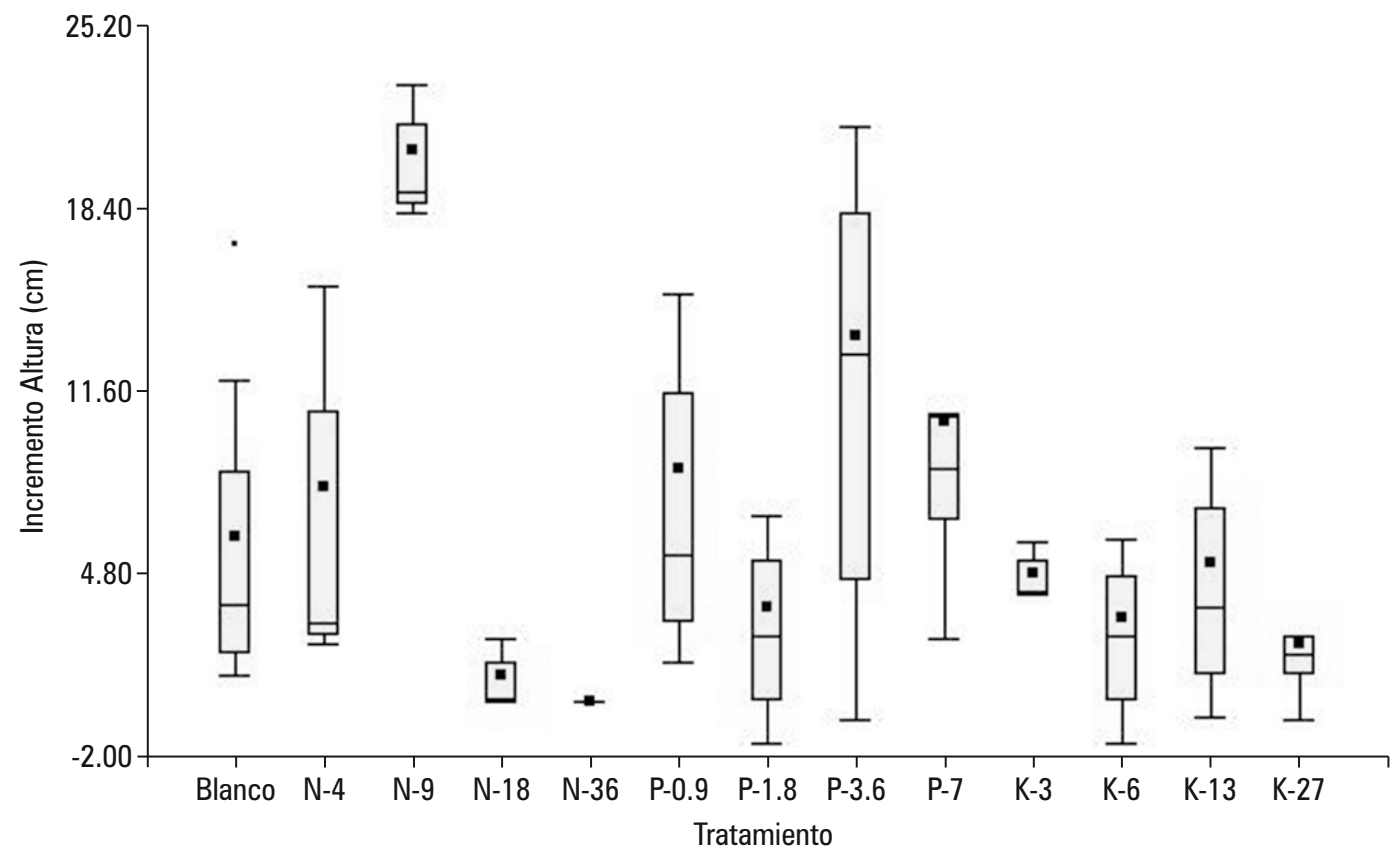

Figura 1. Incremento en altura de plantas de castaña Bertholletia excelsa bajo cuatro niveles de fertilización con N, P y K. 
Tabla 3. Incremento de diámetro basal en plantas de castaña Bertholletia excelsa. Prueba de Tukey.

\begin{tabular}{cccc}
\hline Tratamiento & Medias & $\mathbf{n}$ & Significancia \\
\hline T2 & 0.24 & 3 & A \\
T3 & 0.24 & 3 & A \\
T8 & 0.24 & 3 & A \\
T9 & 0.22 & 3 & A, B \\
T11 & 0.21 & 3 & A, B \\
T1 & 0.19 & 9 & A, B \\
T12 & 0.18 & 3 & A, B \\
T6 & 0.18 & 3 & A, B \\
T10 & 0.13 & 3 & A, B \\
T7 & 0.08 & 3 & A, B \\
T13 & 0.06 & 3 & A, B \\
T4 & 0.03 & 3 & A, B \\
T5 & 0.00 & 3 & B \\
\hline
\end{tabular}

Alfa: $=0.01$ DMS: $=0.23981$

Letras distintas indican diferencias significativas $(p<=0.01)$

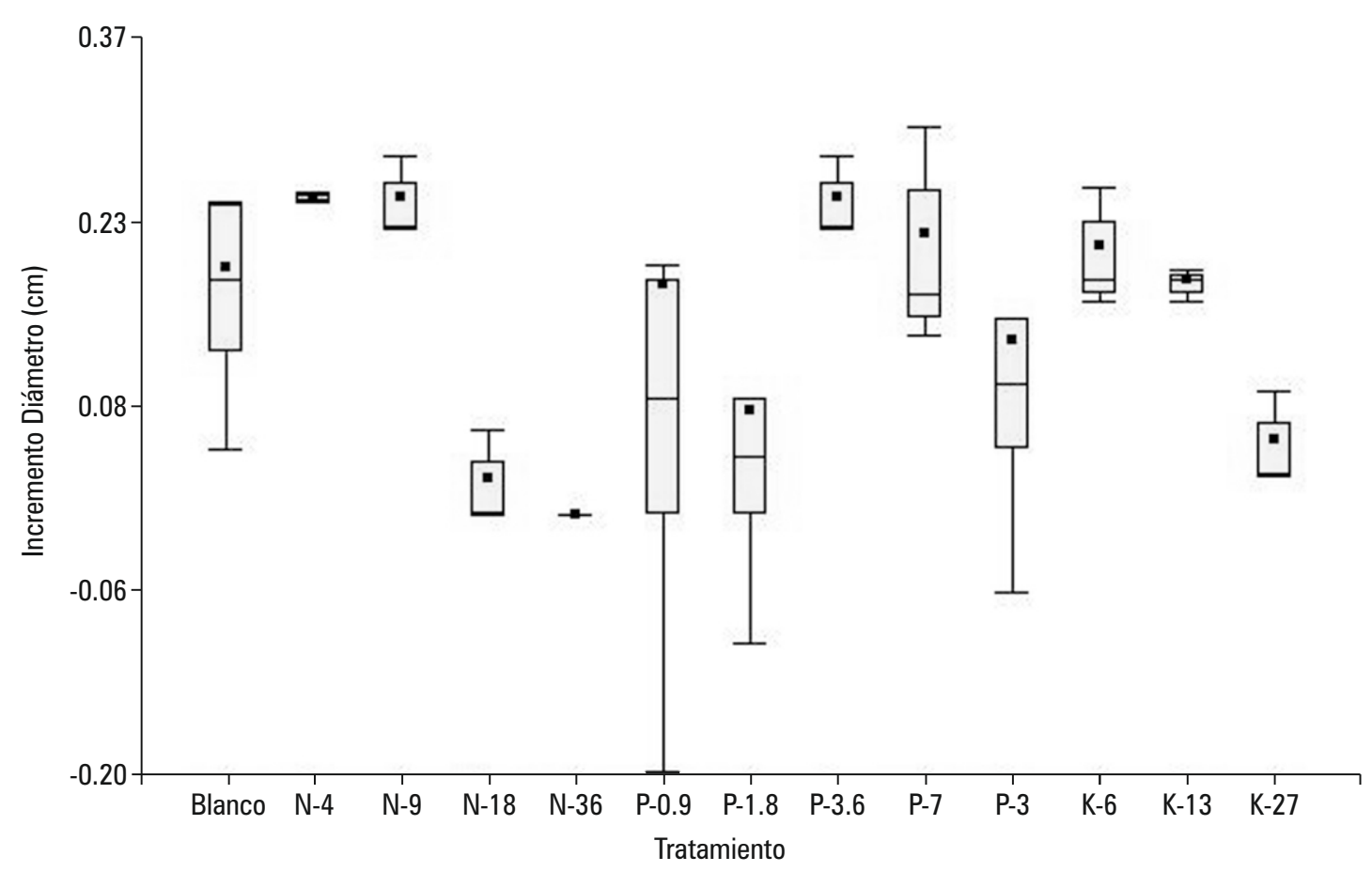

Figura 2. Incremento en diámetro basal de plantas de castaña Bertholletia excelsa bajo cuatro niveles de fertilización con N, P y K. 


\section{DISCUSIÓN}

Generalmente, la respuesta de las plantas de castaña a la fertilización nitrogenada fue alta cuando las dosis fueron intermedias. A medida que la dosis creció, la tasa de asimilación por unidad de fertilizante adicional disminuyó hasta llegar a un valor (dosis crítica) que la planta ya no respondió a los incrementos del fertilizante. Se pone de manifiesto que en la primera etapa del cultivo de la castaña hay una clara respuesta de la planta para la fertilización nitrogenada de hasta $100 \mathrm{~kg} \mathrm{ha}^{-1}$. Emmerich (1997) encontró una clara tendencia en el incremento en la acumulación de biomasa radicular cuando se realizaron aplicaciones de nitrógeno. La estrategia de la especie consiste en una amplia exploración del suelo y formación de un sistema de raíces secundarias para absorber nutrientes de la superficie del suelo.

La respuesta pudiera estar asociada al papel que cumple el nitrógeno en la planta como elemento básico para el normal funcionamiento del metabolismo, formación de compuestos como proteínas, clorofila, enzimas y los procesos de fijación de nitrógeno (Jacob \& Von Vexküll, 1973; Walker et al., 2000) todos relacionados con el crecimiento de la planta y por ende con la producción de biomasa. Asociándose lo anterior a que en el presente estudio se aplicaron diferentes dosis de cada macro elemento, pudiera esperarse una mayor amplitud de la respuesta de esta especie a diferentes combinaciones de dosis y elementos, además de otros factores que pudieran condicionar la respuesta, tales como deficiencias de nutrientes en el suelo, sinergia y/o antagonismo entre los nutrientes.

Bajo las condiciones en las cuales se desarrolló el presente estudio basado en la respuesta a la aplicación de cuatro niveles de fertilización con nitrógeno, fósforo y potasio, localizadas en plantas de Bertholletia excelsa se observó un efecto marcado en la respuesta de las plantas a la fertilización nitrogenada cuando las dosis fueron intermedias ( 50 y $100 \mathrm{~kg} \mathrm{ha}^{-1}$ ). A medida que la dosis creció $\left(200 \mathrm{~kg} \mathrm{ha}^{-1}\right)$ la tasa de asimilación de fertilizante adicional disminuyó hasta llegar al punto que la planta ya no respondió al incremento del fertilizante (dosis crítica) e inclusive a manifestarse un efecto de toxicidad severa cuando se aplicó la dosis mayor (400 $\left.\mathrm{kg} \mathrm{ha}^{-1}\right)$ ocasionando la muerte de las plantas. En los tratamientos que incluyeron dosis de fósforo y potasio los incrementos en las variables evaluadas, generalmente no fueron significativos, exceptuando el tratamiento de $200 \mathrm{~kg} \mathrm{ha}^{-1}$ de fósforo que representó el segundo mejor incremento en altura y en diámetro evaluados (Enmerich, 1997).

\section{BIBLIOGRAFÍA CITADA}

ONERN. 1972. Inventario, evaluación e integración de los recursos naturales de la zona de los ríos Inambari y Madre de Dios. 296p.

Corvera-Gomringer, R.; Del Castillo, D.; Suri, W.; Cusi, E.; Canal, A. 2010. La castaña amazónica (Bertholletia excelsa). Manual de cultivo. Instituto de Investigaciones de la Amazonía Peruana. Madre de Dios. 71p.

Emmerich, S. 1997. Distribution of roots of tropical useful plants in an Agroforestry system tour years alter instalation. In: Annual Report 1997, SHITF-Project ENV 23/2, BMBF $\mathrm{N}^{\circ}$ 0339457B. EMBRAPA. 32-36p.

FAO. 1988. FAO/UNESCO Soil Map of the World, Revised legend, with corrections and updates. World Soil Resources Report 60, FAO, Rome.

Jacob, A.; Von Vexkull, H. 1973. Nutrición y Abonado de los Cultivos Tropicales. $4^{\mathrm{a}}$ Ed. Auroaméricana. 626p.

Kainer, K.; Duryea, M.L.; Macedo, N.C.; Williams, K. 1998. Brazil nut seedling establishment and autoecology in extractive reserves of Acre, Brazil. Ecological Applications, 8 (2): 379410.

Maues, N.M.; Olivera, F.C. 1999. Fenologia reproductiva e entomofauna polinizadora da castanheira-do-Brasil (Bertholletia excelsa Humb. E Bonpl, Lecythidaceae) na amazonia oriental. EMBRAPA/CPATU, 123:25-30.

Myers, G.P.; Newton, A.C.; Melgarejo, O. 2000. The influence of Canopy gap size on natural regeneration of Brazil nut (Bertholletia excelsa) in Bolivia. Forest Ecology and Management, 127:119-128.

Nelson, B.W.; Sabih, B.W. 1985. Observations on flower visitors to Bertholletia excelsa and Coutatari tentuicarpa. Acta Amazonica (Supl.), 15(1/2): 255-234.

O'Malley, D.M.; Buckey, D.P.; Prance, G.T.; Bawa, K.S. 1988. Genetics of Brazil nut (Bertholletia excelsa). Theorical and applied genetics, 76:929-932.

Pardo, M. 2001. Estrutura genética de castanha-dobrasil (Bertholletia excelsa) em floresta e em pastagens do leste do estado do Acre. Disertación de maestría. Escuela Superior de Agricultura Luis de Queirós. 72pp.

Peña-Claros, M.; Boot, R.G.; Zonta, A. 2002. Enrichment planning of Bertholletia excelsa in secondary forest in the Bolivian Amazon: effect of cutting line width on survival, growth and crown traits. Forest Ecology and Management, 161:159-168. 
Peres, C.A.; Baider, C. 1997. Seed dispersal, spatial distribution and population structure of Brazil nut trees (Bertholletia excelsa) in Southeastern Amazonia. Journal of tropical Ecology, 13:6979.

Peres, C.A.; Schiesari, C.; Diaz-Leme, C.D. 1997. Vertebrate predation on Brazil nuts (Bertholletia excelsa), an agouti-dispersed amazonian seed crop: a test of the scape hypothesis. Journal of Tropical Ecology, 13:69-79.

Reátegui-Zirena, E.; Renno, J. F.; Carbajal, F.; Corvera, R.; Del Castillo, D.; García-Dávila, C. 2009. Evaluación de la variabilidad genética de la castaña Bertholletia excelsa en la región Madre de Dios (Perú), mediante marcadores microsatélites.FoliaAmazónica 18(1-2):41-50.
SAS Institute, 2001. User's Guide. Release 8.01. SAS Institute, Cary, NC.

Zuidema, P.A.; Boot, R.G. 2002. Demography of the Brazil nut tree (Bertholletia excelsa) in the Bolivian Amazon: impact of seed extraction on recruitment and population dynamics. Journal of Tropical Ecology, 18:1-13.

Zuidema, P.A.; Dijkman, W.; Rijsoort, J. 1999. Crecimiento de plantines de Bertholletia excelsa en función de su tamaño y la disponibilidad de luz. Ecología en Bolivia, 33:23-35.

Walker, R.L., Burns, I.G., Moorby, J. 2000. Responses of plant growth rate to nitrogen supply: a comparison of relative addition and $\mathrm{N}$ interruption treatments. Journal of Experimental Botany, 52:309-317. 
